Honam Mathematical J. 36 (2014), No. 2, pp. 473-474

http://dx.doi.org/10.5831/HMJ.2014.36.2.473

\title{
ERRATUM TO : "LOCAL SPLITTING PROPERTIES OF ENDOMORPHISM RINGS OF PROJECTIVE \\ MODULES"[HONAM MATH. J. 35(2013), NO.4, PP. 747-755]
}

\section{SANG Cheol Lee}

The following lemma is well-known, but we record it for our references.

Lemma 0.1 ([1], Exercise 33 in Sec.7.3). Let $A$ be a commutative ring with identity. Let $p(x)=a_{0}+a_{1} x+\cdots+a_{n-1} x^{n-1}+a_{n} x^{n}$ be an element of the polynomial ring $A[x]$. Then $p(x)$ is a unit in $A[x]$ if and only if $a_{0}$ is a unit and $a_{1}, a_{2}, \cdots, a_{n}$ are nilpotent in $A$.

Let $A$ be a commutative ring with identity, and let $A[x]$ be a polynomial ring over $A$ with indeterminate $x$. Consider the following

$$
1=(1+x f(x))(1+x g(x))
$$

in $A[x]$. Then we cannot get

$$
\left\{\begin{array}{c}
f(x)+g(x)=0 \\
f(x) g(x)=0
\end{array},\right.
$$

but these two conditions hold if and only if $g(x)=-f(x)$ and $f(x)^{2}=0$. However, since $1+x f(x)$ is a unit in $A[x]$, we can see that $f(x)$ is nilpotent by Lemma 0.1 .

(1) See p.749. $\uparrow$ line $8-\uparrow$ line 5 . The statement "So, we get $\cdots$. From these equations, we can get $f(x)^{2}=0$ " should be replaced by the statement "So, $1+x f(x)$ is a unit in $A[x]$. By Lemma 0.1, $f(x)$ is nilpotent in $A[x]$." Notice that Lemma 2.1 itself in the paper is still true.

(2) See p.753. $\uparrow$ line $14-\uparrow$ line 11. This should be deleted.

(3) See p.751. $\downarrow$ line $7-\downarrow$ line 8 . The statement is not true in general. We can construct a counterexample: Let $s_{1}=s_{2}=1$, and consider $A_{s_{1}}=A_{s_{2}}=A_{s_{1} s_{2}}$. Then $i d \circ \sigma=\sigma=\sigma \circ i d$ and $i d \neq \sigma$. However, the readers may disregard the statement because we do not use it in the later discussions.

Received May 30, 2014. Accepted June 9, 2014. 


\section{References}

[1] David S. Dummit and Richard M. Foote, Abstract Algebra, Prentice-Hall, Inc., 1991.

\section{Sang Cheol Lee}

Department of Mathematics Education and Institute of Pure and Applied Mathematics, Chonbuk National University,

Jeonju, Jeonbuk 561-756, South Korea.

E-mail: scl@jbnu.ac.kr 